THE GRAVITATIONAT FIELDS OF THE MAJOR PLANETS

\author{
S. J. Peale* \\ Department of Physics \\ University of California \\ Santa Barbara, California 93106
}

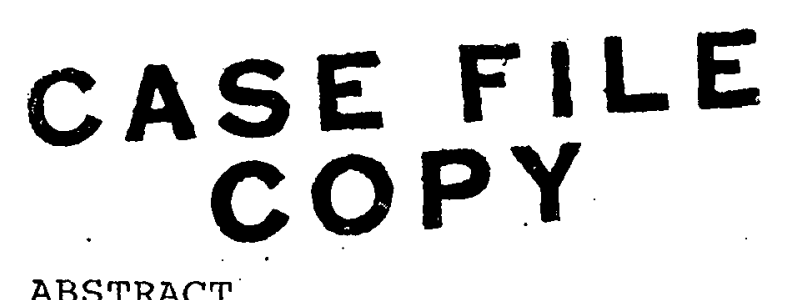

ABSTRACT

The constraints placed on models of the interiors of the major planets by the non-spherical components of their gravitational fields are explained, and several methods of determining these non-spherical components are described and evaluated.

June $1972<$

* Supported in part by the National Aeronautics and Space Administration under Grant NGR 05-010-062. 
Information about the gravitational fields of planetary. . bodies has been obtained either from the observed perturbations of artificial satellites such as those around the moon (Kaula, 1969), the earth (Gaposchkin and Lambeck, 1970), or Mars (Lorell, et al., 1971) or by observations of natural satellites (Brouwer and Clemence, 1961). The latter, either because of limited tracking accuracy or large distance from the planet, have provided information only on the lowest order deviations from spherical symmetry. The artificial satellites on the other hand have defined the gravitational fields much more precisely, primarily because it was possible to closely monitor the perturbations of a wide variety of close orbits. This experience must be extended to the major planets if we are to obtain the details of their external fields. The perturbations of accurately tracked spacecraft are almost the only means of improving our knowledge of these gravitational fields.

The motivation for determining the gravitational fields of the major planets rests in our desire to constrain theoretical models of the interiors. The nature of these constraints and their effect on models of the interior are discussed in section II after some definitions of terms are given. After establishing the motivation for the study, we turn to the actual determination of the harmonic coefficients. Flyby spacecraft are aiscussed in Section III as a first sampling of the field using a least squares 
analysis of the trajectory. The problem of determining the even order zonal harmonics by secular perturbations of orbital elements is defined in section IV, and the magnitudes of the perturbations of an early Jupiter orbiter are given and compared, with tracking accuracies. The necessity for close approaches to major natural satellites and for more than a single orbiter are also shown in this section. The requirement of accurate tracking of additional satellites for higher harmonics leads into a discussion in section $V$ of possible utilization of the Galilean satellites as probes of the gravity field of Jupiter. This will involve radar tracking from the earth. Section VI deals with the tesseral and odd order zonal harmonics and includes reasons why there is likely to be no attempt to measure these coefficients in the reasonably near future. The possibility of a gravity anomaly associated with Jupiter's red spot is discussed in section VII, and a lower bound on a detectable effective mass concentration is estimated. section VIII is a summary and general discussion which ends with a brief paragraph on the alternate technique of a least squares analysis of orbital data. 
II. THE GRAVITATIONAL FIELD AND MODELS

The external gravitational potential of a planetary body is often written

$$
\begin{gathered}
\mathrm{U}=\frac{\mathrm{GM}}{\mathrm{r}}\left[1-\sum_{\ell=2}^{\infty} \mathrm{J}_{\ell} \frac{\mathrm{a}_{e}^{\ell}}{\mathrm{r}^{\ell}} \mathrm{P}_{\ell}(\cos \theta)\right. \\
+\sum_{\ell=2}^{\infty} \sum_{\mathrm{m}=1}^{\ell} \frac{\mathrm{a}_{e}^{\ell} \mathrm{p}_{\ell \mathrm{m}}(\cos \theta)\left[\mathrm{c}_{\ell \mathrm{m}} \cos \mathrm{m} \phi\right.}{\vdots} \\
\left.\left.+\mathrm{s}_{\ell \mathrm{m}} \sin \mathrm{m} \phi\right]\right],
\end{gathered}
$$

where $G$ is the gravitational constant, $M$ is the total mass, $r$ is the separation of the field point from the center of mass of the body, $a_{e}$ is the equatorial radius, $\theta$ and $\phi$ are colatitude and longitude respectively, $\mathrm{P}_{\ell}$ are the Legendre polynomials of order $\ell$ and $P_{\ell m}$ are the associated Legendre functions. The coefficients $J_{\ell}$ and $C_{\ell m}, S_{\ell m}$ are respectively the zonal and tesseral harmonic coefficients, which are determined by differences in moments of the mass distribution about the center of mass. In general

$$
\begin{gathered}
J_{\ell}=-\frac{1}{M a^{\ell}} \iiint_{V} \rho\left(r^{\prime}, \theta^{\prime}, \phi^{\prime}\right) r^{\ell+2} \mathrm{P}_{\ell}\left(\cos \theta^{\prime}\right) \sin \theta^{\prime} \mathrm{d} \theta^{\prime} \mathrm{d} \phi^{\prime} \mathrm{d} r^{\prime} \\
\left\{\begin{array}{l}
C_{\ell m} \\
S_{\ell m}
\end{array}\right\}=-\frac{1}{M a^{l}} \iiint_{V} \rho\left(r^{\prime}, \theta^{\prime}, \phi^{\prime}\right) r^{\prime l+2} \mathrm{P}_{\ell m}\left(\cos \theta^{\prime}\right)\left\{\begin{array}{l}
\cos m \phi \\
\sin m \phi
\end{array}\right\} \sin \theta^{\prime} d \theta^{\prime} d \phi^{\prime} d r^{\prime}
\end{gathered}
$$


where the integration is over the entire volume of the planet. For $\ell=2$

$$
\begin{gathered}
J_{2}=\frac{C-\frac{1}{2} \cdot A-\frac{1}{2} B}{M a_{e}^{2}} \\
\sqrt{c_{22}^{2}+s_{22}^{2}}=\frac{B-A}{4 M a_{e}^{2}}
\end{gathered}
$$

where A, B, C are the principal moments of inertia in the order of increasing magnitude.

If the magnitudes of the harmonic coefficients are determined from the perturbations of natural or artificial satellites of the planet (e.g. Kaula, 1966), Eqs. (2) provide integral constraints on the internal density distribution. For the major planets, these constraints on the density distribution lead to constraints on theoretical models of the interior structure and thus provide the chief motivation for accurate determination of the gravitational fields.

All current models of the interiors of the major planets use the assumption of hydrostatic equilibrium where surfaces of equal density are equipotential surfaces (DeMarcus, 1958; Peebles, 1964; Hubbard, 1969). This is almost certainly a valid assumption for the major planets since their supercritical fluid outer layers can support no static shear stresses. The assumption of hydrostatic equilibrium eliminates all tesseral and odd order zonal harmonics from the expansion of the external field and allows the expression of the even order zonal harmonic 
coefficients as density integrals involving a single parameter or as expansions in the small parameters defining the outer equipotential surface (Peebles, 1964). The harmonic coefficients are uniquely determined by the density distribution which in turn follows from the assumed equation of state, mixing ratio of hydrogen and helium and the temperature distribution.

The procedure then is to calculate a density run for a given set of assumptions, determine the corresponding harmonic coefficients and compare these coefficients with those observed. The agreement between observed and calculated coefficients is a necessary but not a sufficient condition for the correctness of the model. This follows from the fact that the density distribution is not uniquely determined from a finite set of gravity coefficients and drastically different models may produce nearly the same density distribution. This is perhaps best illustrated by the coexistence of the nearly completely solid Jupiter model of DeMarcus (1958) and the completely fluid and uniformly mixed models of Peebles (1964) and Hubbard (1969) which all produce the observed gravitational moments within the rather large errors of observation. At the same time a convective model of Saturn is not consistent with the observed moments, although some of this disagreement may be due to an unknown contribution to the measured coefficients by the rings (Hubbard, 1969). As higher order coefficients depend on higher moments of the mass distribution, the outer layers of the planet 
receive increasing weight in determining a coefficient as the order increases. The sensitivity of the gravity coefficients to the density in the outer layers will make their determination a useful check of the equation of state in the region where it is temperature sensitive and perhaps least understood.

Observations of the natural satellites have provided estimates of $\mathrm{J}_{2}$ and $\mathrm{J}_{4}$. for Jupiter and Saturn and $\mathrm{J}_{2}$ for Neptune (Brouwer and Clemence, 1961). However, $\mathrm{J}_{4}$ for Jupiter is so poorly known that it does not provide a useful constraint on the models (e.g. Peebles, 1964). The more accurate determination of $\mathrm{J}_{2}$ and $\mathrm{J}_{4}$ for the major planets would appear to have first priority to better restrict existing hydrostatic models. However, the high sensitivity of $\mathrm{J}_{6}$ and higher coefficients to changes in the density of the outer layers will make their values extremely useful in defining the equation of state in the nonmetallic regions of the interior.

Although the fluid outer layers of the major planets imply hydrostatic equilibrium, possible deviations from this equilibrium should not be ignored. The detection of nonzero tesseral or odd order zonal harmonics might imply internal convection with dynamic stresses supporting density inhomogeneities. The possibility of a local gravity anomaly in the region of Jupiter's red spot should also not be overlooked.

We shall consider briefly the determination of these terms in the gravitational field expansion which are due to 
non-hydrostatic mass distributions, but the zonal harmonics of even order perhaps deserve greater attention because of their likely complete dominance in the field expansion and their relative ease of unambiguous evaluation through secular perturbations of satellite orbits. 


\section{FLYBY SPACECRAFT.}

The first probes of the gravitational fields of the major planets other than the natural satellites will be flyby spacecraft. It is possible to use rectangular coordinates to describe the motion of the spacecraft and to apply a least squares analysis to the trajectory with $\mathrm{J}_{2}{ }^{\prime} \mathrm{J}_{4}$, the masses and positions of the natural satellites, etc., as unknowns. This technique was applied to the Mariner $V$ flyby of Venus and the range and Doppler tracking to $15 \mathrm{~m}$ and $1 \mathrm{~mm} / \mathrm{sec}$ respectively (Anderson, et al. 1967) allowed the estimate $J_{2}=-5 \pm 10 \times 10^{-6}$ (Anderson and Efron, 1969). Null (1971) has applied a covariance analysis to the Pioneer 10 trajectory past Jupiter which yields probable errors on the determination of $\mathrm{J}_{2}$ and $\mathrm{J}_{4}$ of about $\pm 6 \times 10^{-6}$ and $\pm 10^{-5}$ respectively. Comparison with the current uncertainties given in section IV shows an improvement in the determinations by considerably more than an order of magnitude.

The confidence which one can place in these probable errors depends on a subjective evaluation of the completeness of the model, the possible magnitude of neglected effects, the assignment of weights to the observations and the a priori accuracy initially assumed for the parameters. For example, an unexpectedly large magnetic field interaction or odd order zonal harmonic not included in the model may simulate the effects of $\mathrm{J}_{4}$ during the relatively short interval of close approach to the planet. Still, the least squares analysis of both flyby 
and orbiter trajectories will $"$ provide the first improvements in the estimates of the parameters, and confidence in the values will certainly be sufficient to stimulate a flurry of interior model improvements.

Allowing for the possibility of unanticipated phenomena not included in least squares models, we shall adopt a more conservative approach below which involves secular perturbations of orbiting satellites. Although more involved from a practical point of view, measurements of such secular perturbations will yield values of the even order zonal harmonics which are perhaps less uncertain than those obtained by any other means. 


\section{ARTIFICIAL SATELLITES}

The first artificial satellites of the major planets will likely have highly eccentric orbits with reasonably large semimajor axes. This comes about from the necessity to minimize the velócity change for orbit insertion and from the desire to explore a reasonably large fraction of the near planet environment. It is therefore appropriate that we discuss the effectiveness of such an orbiter in determining the harmonic coefficients of the gravitational field. The need for more than a single satellite will be pointed out where additional coefficients and greater accuracy is desired.

In addition to the magnitudes of the tesseral harmonics of the major planets being small, the rapid rotation of the planets means these terms will lead to high frequency perturbations for the nonresonant, relatively distant satellites considered here. Such perturbations will be negligibly small, and we shall consider here only the even order zonal harmonics. The $J_{2 n}$ coefficients are typically determined from the secular perturbations of longitude of the node $\Omega$ and the longitude of periapsis $\tilde{\omega}$.

$$
\begin{aligned}
\frac{d \Omega}{d t}= & -n \cos i\left[A_{2}(e, i) \frac{a^{2} e^{2} J_{2}}{a^{2}\left(1-e^{2}\right)^{2}}+A_{4}(e, i) \frac{a^{4}}{a^{4}} \frac{J_{4}}{\left(1-e^{2}\right)^{4}}\right. \\
& \left.+A_{6}(e, i) \frac{a^{6} e^{J_{6}}}{a^{6}\left(1-e^{2}\right)^{6}}+\ldots . . .\right]
\end{aligned}
$$




$$
\begin{gathered}
\frac{d \omega}{d t}=n\left[B_{2}(e, i) \frac{a_{e}^{2} J_{2}}{a^{2}\left(1-e^{2}\right)^{2}}+B_{4}(e, i) \frac{a^{4}}{a^{4}} \frac{J_{4}}{\left(1-e^{2}\right)^{4}}\right. \\
\left.+B_{6}(e, i) \frac{a^{6}}{a^{6}} \frac{J_{6}}{\left(1-e^{2}\right)^{6}}+\cdots \cdot \cdot\right] \\
\tilde{\omega}=\omega+\Omega
\end{gathered}
$$

where $\mathrm{n}$ is the mean motion, $a, e, i$ are the semi-major axis, eccentricity and inclination of the orbit relative to the equator plane and the coefficients $A_{i}, B_{i}$ are $0(1)$. The complete literal forms of these coefficients are given by Mueller (1964). The above equations include only the linear perturbations. If $J_{2}=0\left(\frac{\omega^{2} a_{e}^{2}}{G M}\right)$ is considered a small quantity of first order, $\mathrm{J}_{4}=0\left(\mathrm{~J}_{2}^{2}\right) \mathrm{J}_{6}=0\left(\mathrm{~J}_{2}^{3}\right)$ etc., if the planet is in hydrostatic equilibrium. Nonlinear contributions to the secular changes in $\Omega$ and $\omega$ must be included in any solution for the $J_{n}$. However, the linear contribution of the $J_{n}$ with the largest index kept in the above expansions will determine the observational accuracy required for the determination of that coefficient, since the contribution of the nonlinear combinations of lower $J_{n}$ 's should be known with comparable error. For this reason the nonlinear terms will be omitted from the discussion although they must be included in any solution for the coefficients. (See Kozai (1962) or Aksnes (1970) for a literal solution to third order in $\mathrm{J}_{2} \cdot$ ) 
Since the period of tracking the artificial satellite must be relatively short, Eqs. (2) and (3) are not complete in terms higher than first order in $\mathrm{J}_{2}$. The long period terms with arguments involving $w$ must also be included, as short segments of the oscillations will appear as secular contributions. This causes no difficulty, for such terms can be calculated as well as the true secular terms and their effects included in the coefficients of the $\mathrm{J}_{2 \mathrm{n}}$. Tracking through several orbits is anticipated so the short period perturbations, whose amplitudes are small in any case, will average to zero. Whether the contribution of any harmonic to the secular changes in $\omega$ and $\Omega$ is significant or not for a particular orbit depends on the observational errors in determining the positions of the satellite relative to Jupiter, the time spanned by the observations and the certainty with which other perturbations, such as those due to natural satellites, can be eliminated.

These restrictions have limited the present estimates of harmonic coefficients from observations of the natural satellites to $\mathrm{J}_{2}$ and $\mathrm{J}_{4}$ for Jupiter and Saturn and the latter is only known to within about a factor 2 for Jupiter. From the Galilean satellites desitter (1931) finds for Jupiter

$$
\mathrm{J}_{2}=0.01471 \pm .00015
$$

from which Brouwer and Clemence (1961) derive

$$
\mathrm{J}_{4}=-0.00067 \pm .00038
$$


from the observations of the node of $J V$. Since the inner sateliztes of Saturn are much less massive than those of Jupiter, their mutual perturbations are considerably diminished and $\mathrm{J}_{2}$ and $\mathrm{J}_{4}$ are determined with greater accuracy. Jeffreys (1954) finds

$$
\mathrm{J}_{2}=.01667 \pm .00002 \quad \mathrm{~J}_{4}=.00103 \pm .00008 \text {. }
$$

The relatively small errors are qualified by an unknown contribution from Saturn's rings. A recent revision in the radius of Neptune (Freeman and Lynga, 1970) has reduced the value of $\mathrm{J}_{2}$ (Brouwer and Clemence, 1961) to

$$
J_{2}=.0041 \pm .0004
$$

for this planet, and not even $\mathrm{J}_{2}$ is known for Uranus. These uncertainties in size and shape for Uranus and Neptune have frustrated the construction of appropriate models (Newburn and Gulkis, 1971).

If both $\Omega$ and $\omega$ are precisely determined for the artificial satellite (with a sufficiently large orbit eccentricity or inclination) and other perturbations can be removed, the secular equations can be solved for $\mathrm{J}_{2}$ and $\mathrm{J}_{4}$, where $J_{6}$ and subsequent coefficients are assumed zero. The contributions from these neglected terms would be included in the errors for $J_{2}$ and $J_{4}$. Knowledge of $J_{6}$ or higher harmonics necessarily requires perturbations of an independent orbit.

The effects of the high harmonics drop off very rapidly 
as the orbit parameter $p=a\left(1-e^{2}\right)$ increases, such that the orbits of the Galilean satellites are not very. sensitive to the harmonics beyond $\mathrm{J}_{2}$. (On the other hand, if tracking of a single satellite is sufficiently precise and other perturbations are accurately known, the small contributions by higher harmonics for a distant orbit can be used to advantage for a more accurate determination of $\mathrm{J}_{2} \cdot$ )

We shall consider perturbations of satellites of Jupiter as an example. The effects of the first three harmonics on the motion of the nodes of the inner natural satellites and an artificial satellite in a nominal quasi-equatorial orbit with periapsis at $1.1 a_{e}$ and a semi-major axis of $25 a_{e}$ are given in the following table. The values for $\mathrm{J}_{2}$ and $\mathrm{J}_{4}$ were those given above and $J_{6}$ was arbitrarily chosen an order of magnitude less than $\mathrm{J}_{4}$.

Magnitude of Secular perturbations

\begin{tabular}{|c|c|c|c|c|}
\hline . & & $\mathrm{J}_{2}=1.471 \times 10^{-2}$ & $J_{4}=-6.7 \times 10^{-4}$ & $J_{6}=6 \times 10^{-5}$ \\
\hline \multirow{2}{*}{$\begin{array}{l}\text { Artificial } \\
\text { Satellite }\end{array}$} & (node) & $-.116^{\circ} / \mathrm{day}$ & $\begin{array}{r}-7.13 \times 10^{-3} \% \\
\mathrm{day}\end{array}$ & $\begin{array}{r}-7.0 \times 10^{-4} \% / \\
\mathrm{day}\end{array}$ \\
\hline & (periapsis) & .116 & $5.62 \times 10^{-3}$ & $3.7 \times 10^{-4}$ \\
\hline JV & & -2.52 & $-4.40 \times 10^{-2}$ & $-1.1 \times 10^{-3}$ \\
\hline JI & . & $-1.30 \times 10^{-1}$ & $-4.32 \times 10^{-4}$ & $-1.97 \times 10^{-6}$ \\
\hline JII & & $-2.57 \times 10^{-2}$ & $-3.26 \times 10^{-5}$ & $-5.91 \times 10^{-8}$ \\
\hline JIII & . & $-4.98 \times 10^{-3}$ & $-2.48 \times 10^{-6}$ & - \\
\hline JIV & & $-6.93 \times 10^{-4}$ & $-1.12 \times 10^{-7}$ & - \\
\hline
\end{tabular}


For the nearly circular orbits of the natural satellites, the linear terms in $d \tilde{\omega} / d t$ are just the negatives of those for $d \dot{\Omega} / d t$. Mariner $\mathrm{V}$ was tracked to the remarkable precision of less than 15 meters relative to the center of Venus by using both range and range rate information. A Mariner class spacecraft, which would presumably carry the necessary transponder, could be tracked to the same precision in orbit about Jupiter. An orbiting Pioneer without the ranging transponder could be located to within $100 \mathrm{~m}$ in the orbit plane and within perhaps $1 \mathrm{~km}$ in a direction perpendicular to the orbit plane, using Doppler tracking alone (J. D. Anderson, private communication, 1972). From the table, we see that the contribution by $J_{6}$ to the motion of the node and periapsis of the artificial satellite after 100 days is of the order of 0.01 . This corresponds to a $15 \mathrm{~km}$ displacement of the periapsis and a $750 \mathrm{~km}$ displacement of the apoapsis with the displacement of the orbit intersection with the equator plane lying between these two extremes. Hence, the tracking seems certainly adequate to detect perturbations by $J_{6}$ and higher order moments. A possible exception to this conclusion is the case where the orbit is nearly equatorial at the same time the orbit normal is nearly parallel to the plane of the sky. With this exception kept in mind, one might include coefficients higher than $\mathrm{J}_{4}$ in the above equations for the variation in $\Omega$ and $\tilde{\omega}$. However, problems which are independent of tracking must be resolved before accurate values of the coefficients can be obtained.

Including the effects of $J_{6}$ in the secular perturbations of the artificial satellite places a third unknown into the two equations. 
Use of one or more natural satellites (such as JV) to provide additional equations is frustrated by our inability to track these satellites to the same precision as the artificial satellite. In addition, the perturbations due to at least the Galilean satellites and the plasma drag must also be known with errors less than the contribution of $J_{6}$ or less than that of such higher order coefficients which may be desired. The masses of the Galilean satellites are uncertain by as much as $10 \%$, which is the cause of the relatively poor determinations of $\mathrm{J}_{2}$ and $\mathrm{J}_{4}$ (Brouwer and clemence, 1961). In fact, at least one close approach of each of the Galilean satellites by the first Jupiter orbiter should have the highest priority such that these masses can be determined precisely. This alone would improve the values of $\mathrm{J}_{2}$ and $\mathrm{J}_{4}$ from a reanalysis of earth based. observations, and precise masses of the Galilean satellites must be obtained before the full usefulness of the accurate tracking of the artificial satellite can be exploited. For example, suppose the node motion of JV has been precisely determined to provide a third equation for the determination of the coefficients $\mathrm{J}_{2}, \mathrm{~J}_{4}, \mathrm{~J}_{6} \cdot \mathrm{JI}$ (Io) contributes to the precession of the node of JV by the amount

$$
\dot{\Omega}=-\frac{3}{4} \frac{\mathrm{n}_{\mathrm{I}}^{2}}{\mathrm{n}_{\mathrm{V}}^{2}} \frac{\mathrm{m}_{\mathrm{M}}}{\mathrm{M}}=-1.6 \times 10^{-3} \% / \mathrm{day}
$$

But from Eq. (7) and Table $I$ the $10 \%$ uncertainty in the mass of JI leads to a $15 \%$ uncertainty in the contribution of $\mathrm{J}_{6}$ $\left(=6 \times 10^{-5}\right)$ to the node motion of JV. 
The near equatorial orbit necessary for the close approaches of the Galilean satellites makes the node motion of the artificial satellite more difficult to determine. Perhaps the ideal situation would involve an initially equatorial orbit : for satelite mass determinations with a subsequent plane change for ease in following the node. The tracking data for determination of the gravitational moments would be taken over a long period without further encounters of the natural satellites. This scheme would lead to greatly improved values of $\mathrm{J}_{2}$ and $\mathrm{J}_{4}$ ' but $J_{6}$ and higher order coefficients would have to wait for an additional artificial satellite or accurate tracking of the natural satellites to provide the additional equations with sufficient precision for their evaluation.

The above analysis can be transferred to the remaining major planets with some changes. For example, the inner satellites of Saturn are less important because of their much lower masses, whereas the rings pose a unique problem. So little is known about Uranus and Neptune that any artificial satellite would yield much useful information. 


\section{NATURAL SATELLITES}

The natural satellites are existing probes of the gravitational field, and they could provide information on the higher gravitational moments if their motions could be followed closely enough. Television imaging from an artificial satellite is capable of 6 " of arc positional accuracy for a natural satellite against a star background (T. Duxbury, private communication, 1972), and provides one means of defining the orbit perturbations. Whether the accuracy will be sufficiently high depends on the relative positions of the artificial and natural satelites and the number of positional images which can be obtained. A lack of positional accuracy would have to be compensated by a long period of observation, which may exceed the limited spacecraft lifetime. Radio occultations are too infrequent, and any form of bistatic or spacecraft radar too complex for use in tracking the natural satellites (G. L. Tyler, private communication, 1971). However, the Galilean satellites should be within the range of the improved Aericebo radar by 1976, and the necessary precision can be obtained from earth based observations (R. M. Goldstein, private communication, 1971). This technique will be especially effective in combination with a Jupiter orbiter which can provide precise masses independently and thus eliminate the mutual perturbations of the satellites from the equations which determine the gravitational moments. Even $J_{6}$ and $J_{8}$ should be obtainable since 
the lack of sensitivity to these moments is compensated by the precise tracking coupled with arbitrarily long periods of observation. 


\section{DETECTION OF NON-HYDROSTATIC PROCESSES}

The tesseral and odd order zonal harmonics of a planetary gravitational field lead to periodic perturbations of an orbiting' satellite. If they exist at all for the major planets, they are likely to be extremely small and will require that a satellite remain close to the surface for the perturbations to be detectable. In addition to the large velocity change necessary to place a spacecraft in close orbit, the problem. is further complicated by the requirement of several such spacecraft in distinct orbits to sort the coefficients. An infinite series of coefficients which depend on the orbit parameters comprise the amplitude of a given periodic perturbation (e.g. Gaposhkin and Lambeck, 1970). The convergence of this series is minimized as the semi-major axis of the satellite orbit approaches the planetary radius. Thus the penalty of high sensitivity to the higher order harmonics is the uncertainty of the relative contributions of the harmonics to a given amplitude. This uncertainty is resolved with perturbations of several distinct orbits which have distinct series for the amplitude of a given periodic perturbation. The solution of a set of simultaneous algebraic equations then yields the coefficients (Gaposhkin and Lambeck, 1970). The requirement of many close orbiters for the determination of the tesseral and odd zonal harmonics of the major planets reinforces our natural tendency to assume them negligible. 


\section{GRAVITY ANOMALIES}

One of the significant results of tracking the lunar orbiters was the discovery of near surface gravity anomalies (Muller'and Sjogren, 1968). Jupiter's red spot may be a Taylor column (Hide, 1963, 1969) above a hydrogen iceberg floating in a region of rapid density increase of a hydrogen-helium fluid (Streett, 1969). A spacecraft flying over such an iceberg would sense a positive gravity anomaly, since the lower density solid hydrogen is closer to the spacecraft than the displaced equal mass of fluid would be in the absence of the iceberg. If a spacecraft were flying over a gravity anomaly while it was on the limb of Jupiter as viewed from the earth, the amplitude of the line of sight velocity residual from a calculated orbit is given by

$$
\Delta v=\int_{0}^{t} a / / d t=\int_{r_{\text {min }}}^{r} \frac{G \Delta M}{r^{\prime 2}} \cos \alpha \frac{d r^{\prime}}{v}
$$

where $t=0$ is the time of closest approach $a / /$ is the acceleration parallel to the line of sight, $\Delta M$ is the effective mass causing the gravity anomaly $r$ is the separation between $\Delta M$ (here assumed a point mass) and the spacecraft, $\alpha$ is the angle between the direction to $\Delta M$ and the spacecraft velocity (here assumed parailel to the line of sight), $v$ is the relative velocity between the spacecraft and the gravity anomaly. For the elliptic orbit considered above $r_{\min } \approx 0.1 \mathrm{a}_{\mathrm{e}}$ and $\mathrm{v} \approx 5 \times 10^{6} \mathrm{~cm} / \mathrm{sec}$. With 
the upper bound on the integration $r=0.2 \mathrm{a}_{\mathrm{e}}$, we have

$$
\Delta v=3.8 \times 10^{-24} \Delta M
$$

If the minimum $\Delta v$ detectable is $1 \mathrm{~mm} / \mathrm{sec}$, the lower bound.

on the fractional mass detectable as a point mass concentration near the surface is

$$
\frac{\Delta \mathrm{M}}{\mathrm{M}} \approx 10^{-8}
$$

This limit assumes that the orbit would be precisely known in the absence of the anomaly, so in actual practice the minimum $\Delta \mathrm{M}$. detectable will be considerably larger. 
VIII. DISCUSSION

The chief motivation for obtaining accurate values of the even order zonal harmonic coefficients of the expanded gravitational fields of the major planets is the constraint these coefficients place on the internal mass distributions and hence on theoretical models of the planetary interiors. Although the values of a finite number of coefficients cannot define a model or prove a given one correct, a model which does not produce the values of these coefficients is known to be incorrect. The composition and temperature distributions and the equations of state may be better defined if certain combinations produce incorrect models. The higher order coefficients are especially sensitive to changes in density in the outer regions of the planets where the equation of state is temperature sensitive and will therefore be useful in the discussion of models of the extended, supercritical atmospheres. The values of $\mathrm{J}_{2}$ and $\mathrm{J}_{4}$ and other data for Uranus and Neptune, which are only obtainable with artificial satellite observations, may allow meaningful model construction for these planets.

The secular perturbations of a single artificial satellite in an eccentric orbit will yield values of $\mathrm{J}_{2}$ and $\mathrm{J}_{4}$ with an error determined by the contribution of the neglected $J_{6}$ and higher terms provided the secular perturbations from other sources are known with similar precision. This is especially 
true of the Jupiter system where better masses of the Galilean satellites must be determined by close approaches of the artificial satellite before much improvement in $J_{2}$ and $J_{4}$ can be realized. The better masses of the Galilean satellites alone will improve the values of $\mathrm{J}_{2}$ and $\mathrm{J}_{4}$ by reanalysis of the old observations. The determination of $\mathrm{J}_{6}$ and higher order coefficients must await the precise tracking of additional satellites. In the case of Jupiter these satellites may be the Galilean satellites tracked by earth-based radar.

Nonzero tesseral and odd order zonal harmonics would indicate internal dynamics. However, attempts to determine them would demand the high price of many close orbiters and reasonable upper bounds on their values might be obtained only in the distant future.

Near surface mass concentrations are unlikely in the fluid outer layers of the major planets, but one may be associated with the Jupiter's red spot. The estimated absolute lower bound on the detectable effective mass of such an anomaly yields $\Delta M / M=10^{-8}$.

The rectangular coordinates rather than the orbital elements can be used to describe the motion of the artificial satellite and a least squares analysis applied with $\mathrm{J}_{2}, \mathrm{~J}_{4}$, the masses of the natural satellites, etc., as unknowns. The small probable errors in the parameters, even for a flyby spacecraft, indicate the power of this method with current tracking accuracies. When applied to a single orbiter with high 
orbit eccentricity, the least squares analysis may yield probable errors small enough to estimate $J_{6}$, the probable errors in the satellite masses also being reduced in the analysis (J. D. Anderson, private communication, 1972). However, the success of such an approach depends on the a priori accuracy assumed for the various parameters at the beginning, the subjective assignment of weights to the observations and the completeness of the model. We justified above the neglect of the tesseral harmonics and zonal harmonics of odd order, but we have been surprised in the past by the fallacy of such reasonable arguments. Since the secular changes in $\Omega$ and $\omega$ depend only on the zonal harmonics of even order in the gravity field of Jupiter, the determination of these motions over as long a time scale as possible is perhaps less uncertain than the least squares analysis. A combination of the two methods has worked to great advantage in determining the harmonic coefficients of the earth's field. 
REFERENCES

1. Aksnes, K.: 1970, Jet Propulsion Laboratory, California . Institute of Technology Technical Report 32-1507.

2. Anderson, J. D., Pease, G..E., Efron, L., Tausworthe, R. C.: 1967, Science $158,1689$.

3. Anderson, J. D., and Efron, L.: 1969, (A), Bull. Amer. Astron. Soc. 1,231 .

4. Brouwer, D., and Clemence, G. M.: 1961, in the Solar system Vol. III, Planets and Satellites, G. P. Kuiper and B. M. Middlehurst, Ed. Univ of Chicago Press, pp. 31-94.

5. DeMarcus, W. C.: 1958, Astron. J. 63, 2 .

6. Freeman, K. C., and Lynga, G.: 1970, Astrophys. J. 160, 767.

7. Gaposchkin, E. M., and Lambeck, K.: 1970, Smithsonian Astrophysical observatory, SR 315 .

8. Hide, R.: 1963, Mem. Soc. Roy. Sci. Liegé 7, 481.

9. Hide, R.: 1969, J. Atmos. Sci. 26, 841 .

10. Hubbard, W. B.: 1969, Astrophys. J. 155, 333.

11. Jeffreys, H.: 1954, Mon. Nat. Roy. Ast. Soc. 114, 433.

12. Kaula, W. M.: 1966, Theory of Satellite Geodesy, Blaisdell Publishing Co., Waltham, Mass.

13. Kaula, W. M.: 1969, Science 166, 1581.

14. Kozai, Y.: 1962, Astron. J. 67, 446 .

15. Lorell, J., Born, G. H., Christensen, E. J., Jordan, J. F., Laing, P. A., Martin, W. L., Sjogren, W. L., Shapiro, I. I., Reasenberg, R. D., and Slater, G. L.: 1972, Science 175, 317. 
16. Mueller, I. I.: 1964, Introduction to Satellite Geodesy, Frederick Ungar Publishing Co., New York.

17. Muller, P. M. and Sjogren, W. L.: 1968, Science 161, 680 .

18: Newburn, R. L., Jr., and Gulkis, S.: 1971, Jet Propulsion Laboratory, California Institute of Technology, Technical Report 32-1529.

19. Nul1, G. W.: 1971, Jet Propulsion Laboratory Report TM 391-221.

20. Peebles, P. J. E.: 1964, Astrophys. J. 140, 328.

21. Sitter, W. de: 1931, Mon. Nat. Roy. Ast. Soc. 91, 706.

22. Streett, W. B.: 1969, J. Atmos. Sci. 26, 924. 